\title{
Beta cell compensation for insulin resistance in Zucker fatty rats: increased lipolysis and fatty acid signalling
}

\author{
C. J. Nolan • J. L. Leahy • V. Delghingaro-Augusto • \\ J. Moibi • K. Soni • M.-L. Peyot • M. Fortier • C. Guay • \\ J. Lamontagne $\cdot$ A. Barbeau $\cdot$ E. Przybytkowski • \\ E. Joly • P. Masiello $\cdot$ S. Wang $\cdot$ G. A. Mitchell • \\ M. Prentki
}

Received: 15 January 2006 / Accepted: 11 April 2006 / Published online: 26 July 2006

(C) Springer-Verlag 2006

\begin{abstract}
Aims/hypothesis The aim of this study was to determine the role of fatty acid signalling in islet beta cell compensation for insulin resistance in the Zucker fatty $f a / f a(Z F)$ rat, a genetic model of severe obesity, hyperlipidaemia and insulin resistance that does not develop diabetes.

Materials and methods NEFA augmentation of insulin secretion and fatty acid metabolism were studied in isolated islets from ZF and Zucker lean (ZL) control rats.

Results Exogenous palmitate markedly potentiated glucosestimulated insulin secretion (GSIS) in ZF islets, allowing robust secretion at physiological glucose levels $(5-8 \mathrm{mmol} / \mathrm{l})$.
\end{abstract}

Electronic supplementary material Supplementary material is available for this article at http://dx.doi.org/10.1007/ s00125-006-0305-5 and is accessible for authorized users.

C. J. Nolan $\cdot$ V. Delghingaro-Augusto $\cdot$ M.-L. Peyot $\cdot$ C. Guay $\cdot$

J. Lamontagne $\cdot$ A. Barbeau $\cdot$ E. Przybytkowski $\cdot$ E. Joly $\cdot$

P. Masiello $\cdot$ M. Prentki

Molecular Nutrition Unit and Montreal Diabetes Research Center,

University of Montreal and Centre Hospitalier

de l'Université de Montréal,

Montreal, QC, Canada

J. L. Leahy $\cdot$ J. Moibi

Division of Endocrinology, Diabetes and Metabolism,

University of Vermont,

Burlington, VT, USA

K. Soni $\cdot$ M. Fortier $\cdot$ S. Wang $\cdot$ G. A. Mitchell

Division of Medical Genetics, Sainte-Justine Hospital,

Montreal, QC, Canada

C. J. Nolan $(\bowtie)$

Department of Endocrinology, Canberra Hospital and Medical School, Australian National University,

P.O. Box 11, Woden, ACT 2606, Australia

e-mail: christopher.nolan@anu.edu.au
Exogenous palmitate also synergised with glucagon-like peptide-1 and the cyclic AMP-raising agent forskolin to enhance GSIS in ZF islets only. In assessing islet fatty acid metabolism, we found increased glucose-responsive palmitate esterification and lipolysis processes in ZF islets, suggestive of enhanced triglyceride-fatty acid cycling. Interruption of glucose-stimulated lipolysis by the lipase inhibitor Orlistat (tetrahydrolipstatin) blunted palmitate-augmented GSIS in ZF islets. Fatty acid oxidation was also higher at intermediate glucose levels in ZF islets and steatotic triglyceride accumulation was absent.

Conclusions/interpretation The results highlight the potential importance of NEFA and glucoincretin enhancement of insulin secretion in beta cell compensation for insulin resistance. We propose that coordinated glucose-responsive fatty acid esterification and lipolysis processes, suggestive of triglyceride-fatty acid cycling, play a role in the coupling mechanisms of glucose-induced insulin secretion as well as in beta cell compensation and the hypersecretion of insulin in obesity.

Keywords Fatty acid partitioning · Fatty acid signalling · Glucagon-like peptide $1 \cdot$ Insulin resistance $\cdot$ Insulin secretion - Islet beta cell compensation - Lipolysis . Triglyceride-fatty acid cycling $\cdot$ Zucker fatty rat

$\begin{array}{ll}\text { Abbreviations } \\ \text { ACACA } & \text { acetyl-CoA carboxylase } \alpha \\ \text { ACACB } & \text { acetyl-CoA carboxylase } \beta \\ \text { CPT1 } & \text { carnitine palmitoyltransferase-1 } \\ \text { ESM } & \text { Electronic Supplementary Material } \\ \text { FFAR1 } & \text { free fatty acid receptor 1 } \\ \text { FASN } & \text { fatty acid synthase } \\ \text { GLP-1 } & \text { glucagon-like peptide-1 } \\ \text { Gly-3-P } & \text { glycerol-3-phosphate }\end{array}$


GPAM mitochondrial glycerol-3-phosphate acyltransferase

GSIS glucose-stimulated insulin secretion

HSL hormone-sensitive lipase

LC-CoA long chain acyl-CoA

NCEH neutral cholesteryl ester hydrolase

PKA cyclic AMP-dependent protein kinase

PPARA peroxisomal proliferator activator receptor- $\alpha$

SREBF1C sterol-regulatory element binding factor 1c

TG triglyceride

TG-FA triglyceride-fatty acid

TGL triglyceride lipase

ZF Zucker fatty

ZL Zucker lean

\section{Introduction}

Normoglycaemia is maintained in the majority of obese insulin-resistant individuals, as their islet beta cells compensate for insulin resistance with insulin hypersecretion [1, 2]. Obesity-associated type 2 diabetes develops when beta cell compensation processes fail $[3,4]$. The mechanisms of islet beta cell compensation, however, are poorly understood. Particularly unclear is the role of excess circulating NEFA, which frequently coexists with insulin resistance. Acutely elevated NEFA are well known to enhance glucose-stimulated insulin secretion (GSIS) [3, 5]; however, some investigators believe that persistently elevated NEFA may be detrimental to beta cell function or viability $[6,7]$. We have previously utilised the Zucker $f a / f a$ fatty $(\mathrm{ZF})$ rat model of insulin resistance in order to study beta cell compensation [8] and have done so again for the present work, which focuses on the role of NEFA. The ZF rat has a mutated leptin receptor and, as a consequence, is hyperphagic, obese, hyperlipidaemic and severely insulin-resistant [8-10]. Importantly, unlike the Zucker diabetic fatty rat, it does not develop diabetes [8-10], which makes it a good model for the investigation of islet beta cell compensation for insulin resistance.

In earlier work, we reported increased islet glucose utilisation and oxidation in $\mathrm{ZF}$ compared with control Zucker lean (ZL) islets [8]. Increased glucose oxidation should contribute to the compensatory hyperinsulinaemia in $\mathrm{ZF}$ rats by increasing the activity of the $\mathrm{K}_{\mathrm{ATP}}$ channel/ $\mathrm{Ca}^{2+}$ pathway of nutrient-insulin secretion coupling [11]. We also showed increased activities of enzymes and increased metabolites of the anaplerotic and malate-pyruvate cycling pathways [8]. As anaplerosis is the pathway by which glucose increases malonyl-CoA levels, these results led us to hypothesise that enhanced glucose-induced increases in malonyl-CoA/long chain acyl-CoA (LC-CoA) signalling may also be involved in $\mathrm{ZF}$ islet compensation. This is based on the malonyl-CoA/LC-CoA model of lipid signalling for insulin secretion that we have described previously $[12,13]$. Central to the model is that an increase in glucose, via increasing malonyl-CoA, which inhibits carnitine palmitoyltransferase-1 (CPT1), as was first described by McGarry and Brown [14], switches the partitioning of NEFA in the beta cell away from oxidation towards the accumulation of LC-CoA and fatty acid acylation and esterification products (e.g. diacylglycerol [3, 13-15]), which are proposed to have direct regulatory effects on insulin secretion $[3,12,13]$.

Lipolysis is known to be active in the beta cell, and has been implicated in both lipid signalling for insulin secretion [16-18] and, if dysregulated, beta cell failure [19]. However, little is known about its regulation and which lipolytic enzymes are operative. A question addressed in this study was whether activity of the lipolysis arm of beta cell lipid partitioning might be enhanced in situations of chronically increased malonyl-CoA/LC-CoA signalling as a way of protecting islets from excess lipid accumulation $[3,20]$.

The present study focused on the role of lipid signalling in beta cell compensation in ZF rats. The effects of NEFA in augmenting GSIS, alone and in combination with the incretin hormone glucagon-like peptide-1 (GLP-1), and the major pathways of intracellular fatty acid partitioning, including lipolysis, were studied in isolated islets from $\mathrm{ZF}$ and ZL control rats.

\section{Materials and methods}

Animals and measurement of blood and plasma parameters

Zucker fatty ( $f a / f a, Z F)$ and Zucker lean $(f a /+$ or $+/+, \mathrm{ZL})$ rats (Harlan, Indianapolis, IN, USA) were fed standard laboratory chow and were all studied at 10 weeks of age. All protocols were approved by the Institutional Animal Care and Use Committee of the University of Vermont. Tail vein blood sampling was performed between 08.00 and $09.00 \mathrm{~h}$ from non-anaesthetised, fed rats for measurement of blood glucose using a glucose monitor (Therasense, Alameda, CA, USA), plasma insulin (Insulin RIA kit; Linco Research, St Charles, MS, USA), plasma triglyceride (TG) (GPO Trinder; Sigma-Aldrich, Saint Louis, MS, USA) and plasma NEFA (NEFA C kit; Wako Chemicals, Neuss, Germany).

Islet isolation and culture

Fed rats were anaesthetised between 08.00 and $09.00 \mathrm{~h}$ with sodium pentobarbital and then killed by exsanguination, and pancreatic islets were isolated as described previously 
[21]. Prior to initiating experiments or collecting islets for analysis, islets were rested for $1 \mathrm{~h}$ in regular RPMI 1640 medium supplemented with $10 \%$ fetal calf serum, $10 \mathrm{mmol} / 1$ HEPES ( $\mathrm{pH} 7.4), 1 \mathrm{mmol} / \mathrm{l}$ sodium pyruvate, $100 \mathrm{U} / \mathrm{ml}$ penicillin and $100 \mu \mathrm{g} / \mathrm{ml}$ streptomycin (RPMI complete) at $11 \mathrm{mmol} / 1$ glucose at $37^{\circ} \mathrm{C}$ in a humidified atmosphere containing $5 \% \mathrm{CO}_{2}$.

Measurement of islet insulin secretion

Insulin secretion was measured in freshly isolated islets from $\mathrm{ZL}$ and $\mathrm{ZF}$ rats at various concentrations of glucose (3$16 \mathrm{mmol} / \mathrm{l})$ and palmitate $(0-0.4 \mathrm{mmol} / \mathrm{l})$ in the absence or presence of $10 \mathrm{nmol} / 1 \mathrm{GLP}-1,5 \mu \mathrm{mol} / 1$ forskolin or $0.2 \mathrm{mmol} / 1$ Orlistat (tetrahydrolipstatin), as described in detail in the Electronic Supplementary Material (ESM). Total insulin content of the islets was measured following extraction with acid-ethanol $(0.2 \mathrm{mmol} / \mathrm{l} \mathrm{HCl}$ in $75 \%$ ethanol $)$.

Determination of islet DNA, protein and triglyceride content

Batches of 15 freshly isolated islets were used for determination of DNA and protein as described previously [18], and batches of 75-100 freshly isolated islets were used for determination of TG [18].

Oxidation and esterification of islet fatty acids

Fatty acid oxidation and esterification were measured in isolated islets of $\mathrm{ZL}$ and $\mathrm{ZF}$ rats using radiolabelled tracers, as described in detail in the ESM.

Measurement of islet lipolysis

Lipolysis was measured in freshly isolated islets from $\mathrm{ZL}$ and $\mathrm{ZF}$ rats as the rate of glycerol release at 3 and $16 \mathrm{mmol} / 1$ glucose in the presence or absence of $10 \mathrm{nmol} / \mathrm{l} \mathrm{GLP}-1,5 \mu \mathrm{mol} / 1$ forskolin or $0.2 \mathrm{mmol} / 1$ Orlistat, as described in detail in the ESM.

Assays for activities of triglyceride lipase and neutral cholesteryl ester hydrolase

Triglyceride lipase (TGL) and neutral cholesteryl ester hydrolase (NCEH) enzyme activities were determined in islet homogenates from ZL and ZF islets, as described in the ESM.

RNA extraction and RT-PCR analysis

RT-PCR analyses of ZF and ZL islets were performed according to methods previously described [21], as detailed in the ESM.
Statistical analysis

All results are expressed as mean \pm SEM. Statistical significance was calculated with Student's $t$-test or, for multiple comparisons, two-way ANOVA with Bonferroni post hoc testing as indicated. A $p$ value of $<0.05$ was considered significant. Prism v.4 (GraphPad Software, San Diego, CA, USA) was used to perform the analyses.

\section{Results}

Body weight, fed blood chemistry at $09.00 \mathrm{~h}$ and islet parameters

Compared with ZL rats, ZF rats were markedly obese, with severe hypertriglyceridaemia, moderately elevated NEFA levels and a greater than 10-fold increase in insulinaemia (Fig. 1a,c-e). Blood glucose, however, was normal (Fig. 1b). These results are consistent with our previous findings $[8]$ and those of others $[9,10]$ in the non-diabetic ZF rat, confirming that, in this model, markedly enhanced insulin secretion compensates for the obesity-associated insulin resistance without evidence of beta cell failure. Islets from $\mathrm{ZF}$ rats were 2- to 2.5 -fold larger than $\mathrm{ZL}$ islets according to total protein and DNA contents (Fig. 1f,g). TG content, expressed per $\mathrm{mg}$ of islet protein, however, was $19 \%$ lower in ZF compared with ZL islets (Fig. 1h). Insulin content, expressed per mg protein, was $43 \%$ lower in $\mathrm{ZF}$ compared with ZL islets (Fig. 1i).

Augmentation of insulin secretion by exogenous NEFA is enhanced in $\mathrm{ZF}$ islets

In the absence of exogenous palmitate, GSIS was increased in ZF compared with ZL islets $\sim 5$-fold at both 8 and $16 \mathrm{mmol} / 1$ glucose $(p<0.001)$ (Fig. $1 \mathrm{j})$. Exogenous palmitate at $0.2 \mathrm{mmol} / \mathrm{l}$ augmented GSIS at $16 \mathrm{mmol} / \mathrm{l}$ glucose by $38 \%$ in $\mathrm{ZF}$, but had no effect in ZL islets (Fig. 1j). Exogenous palmitate at the higher concentration of $0.4 \mathrm{mmol} / \mathrm{l}$ augmented GSIS at 5,8 and $16 \mathrm{mmol} / \mathrm{l}$ glucose (8-, 4- and 3-fold, respectively) in ZF islets, but in ZL islets increased GSIS by 3 -fold at $16 \mathrm{mmol} / \mathrm{l}$ glucose only (Fig. 1j). Figure 1k magnifies the insulin secretion data at $5 \mathrm{mmol} / \mathrm{l}$ glucose shown in Fig. 1j. Clearly, exogenous palmitate allowed very robust insulin secretion at a physiologically low $(5 \mathrm{mmol} / \mathrm{l})$ glucose concentration in ZF islets only. Hence, ZF compared with ZL islets are more sensitive to the effect of exogenous NEFA in augmenting GSIS. Furthermore, the glucose set-point for insulin secretion is lowered by exogenous NEFA in ZF, but not $\mathrm{ZL}$, islets. 

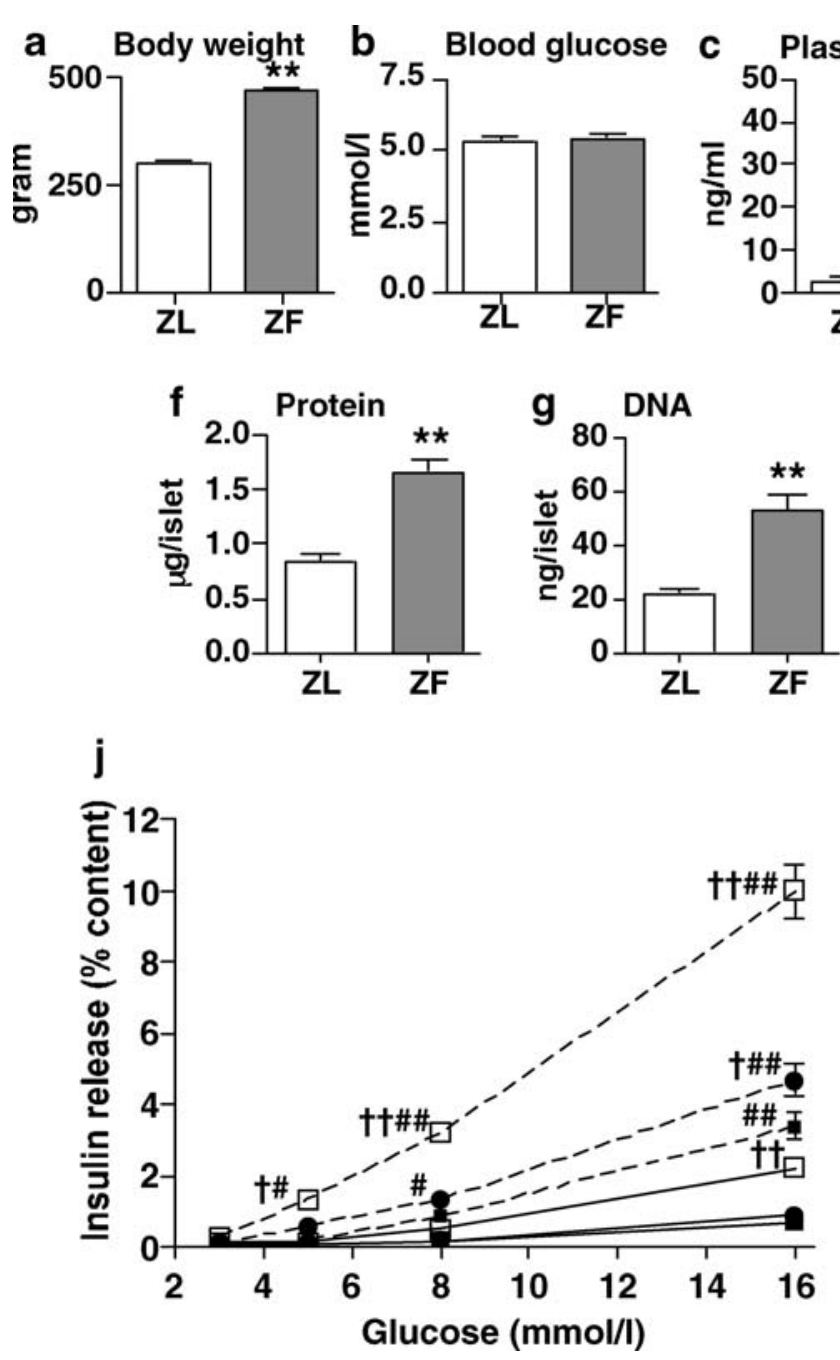

Fig. 1 NEFA augmentation of glucose-stimulated insulin secretion is markedly enhanced in ZF rat islets. a Body weight of 10-week-old ZL and $\mathrm{ZF}$ rats. Means \pm SEM of 24 rats per group. ${ }^{* *} p<0.001$ compared with ZL. b-e Blood glucose, plasma insulin, plasma triglyceride (TG) and plasma NEFA, respectively, in 10-week-old ZL and ZF rats fed at $09.00 \mathrm{~h}$. Means \pm SEM of $6-20$ rats per group. ${ }^{*} p<0.05,{ }^{* *} p<0.001$ compared with ZL. f-i ZL and ZF islet protein, DNA, TG and insulin contents. Means \pm SEM of islets from $5-24$ rats per group. ${ }^{*} p<0.01$, ${ }^{* *} p<0.001$ compared with ZL. $\mathbf{j}$ Insulin secretion in islets from ZF

GLP-1 and forskolin synergise with NEFA to augment GSIS in ZF rats

The effects of $10 \mathrm{nmol} / \mathrm{l} \mathrm{GLP}-1$ and $5 \mu \mathrm{mol} / \mathrm{l}$ forskolin in augmenting GSIS in the absence and presence of $0.25 \mathrm{mmol} / 1$ palmitate were assessed in islets of $\mathrm{ZL}$ and ZF rats (Fig. 2). GLP-1, palmitate, and the combination of both, had no effect on insulin secretion at $3 \mathrm{mmol} / \mathrm{l}$ glucose in either ZL or ZF islets (Fig. 2a). Lack of effect of GLP-1 and palmitate, alone or in combination, on GSIS was also found at $8 \mathrm{mmol} / \mathrm{l}$ glucose in $\mathrm{ZL}$ islets (Fig. 2b). In ZF islets at $8 \mathrm{mmol} / \mathrm{l}$ glucose, however, palmitate increased GSIS 1.7-fold, GLP-1 increased GSIS 2.6-fold, and
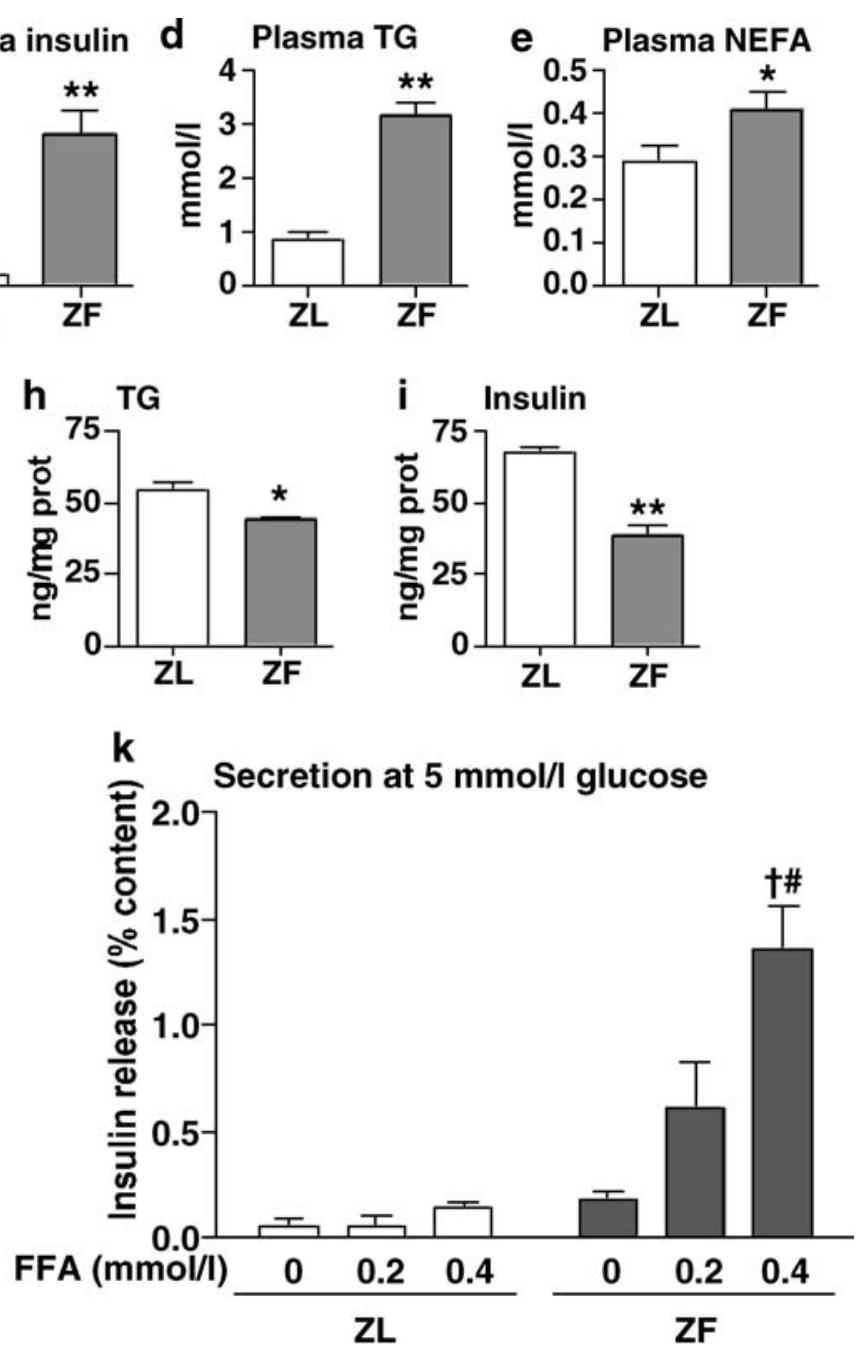

(dashed lines) and ZL (full lines) rats incubated at 3, 5, 8 or $16 \mathrm{mmol} /$ 1 glucose in the presence of 0 (closed squares), 0.2 (closed circles) or 0.4 (open squares) $\mathrm{mmol} / \mathrm{l}$ palmitate. $\mathbf{k}$ Secretion values at $5 \mathrm{mmol} /$ 1 glucose. Means \pm SEM $(\mathbf{j}, \mathbf{k})$ of six determinations from islets of six rats of each genotype in two separate experiments. Two-way ANOVA post hoc analyses: $\# p<0.01, \# \# p<0.001$ compared with $\mathrm{ZL}$ for the same condition; $\uparrow p<0.01, \uparrow \uparrow p<0.001$ compared with the condition of no palmitate for the same genotype

palmitate together with GLP-1 dramatically increased GSIS by 8.8 -fold (Fig. 2b). Thus, ZF islets have increased sensitivity to the incretin effect of GLP-1, and this effect is markedly augmented in the presence of palmitate, consistent with synergism in action between GLP-1 and NEFA. Forskolin, an activator of adenylate cyclase that increases islet cyclic AMP levels, was even more potent than GLP-1 in augmenting insulin secretion. Whereas GLP$1 \mathrm{had}$ no effect on insulin secretion at $3 \mathrm{mmol} / 1$ glucose in $\mathrm{ZL}$ and $\mathrm{ZF}$ islets, forskolin did increase insulin secretion in ZF islets (2.5-fold), an effect increased by palmitate (a further 2.5-fold) (Fig. 2a). At $8 \mathrm{mmol} / 1$ glucose, forskolin dramatically increased GSIS in ZF islets (8.4-fold), and this 

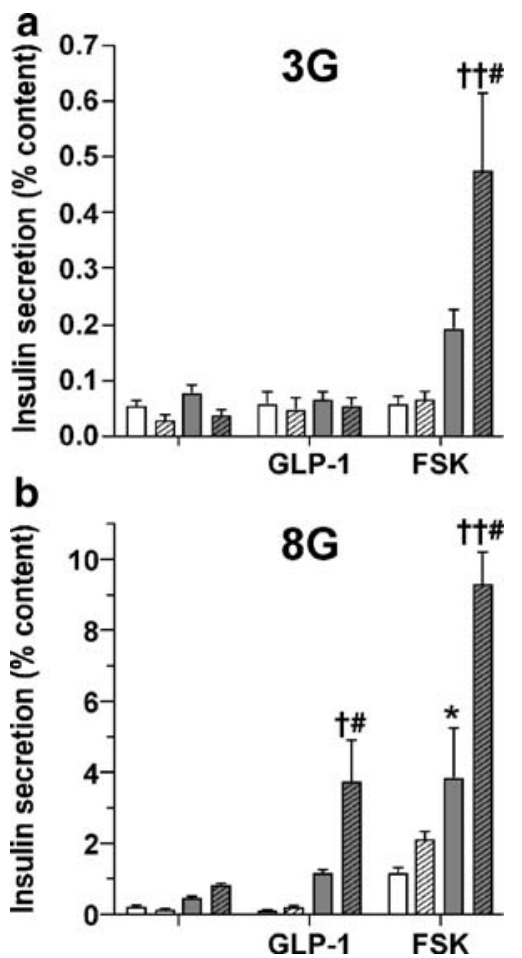

Fig. 2 NEFA synergise with GLP-1 and forskolin to enhance glucosestimulated insulin secretion in compensating ZF islets. a, b Insulin secretion in islets from ZF (grey bars) and ZL (white bars) islets incubated at (a) $3 \mathrm{mmol} / 1$ glucose and (b) $8 \mathrm{mmol} / \mathrm{l}$ glucose in the absence (non-hatched bars) and presence (hatched bars) of $0.25 \mathrm{mmol} / 1$ palmitate and $10 \mathrm{nmol} / 1 \mathrm{GLP}-1$ or $5 \mu \mathrm{mol} / 1$ forskolin (FSK). Means \pm SEM of six determinations from islets of six rats of each genotype in two separate experiments. Two-way ANOVA post hoc analyses: ${ }^{*} p<0.01, \# p<0.001$ compared with $\mathrm{ZL}$ for the same condition; $\dagger p<0.05$, $\dagger \uparrow p<0.001$ compared with $\mathrm{ZF}$ for the same condition in the absence of palmitate

effect was increased by palmitate (a further 2.4-fold) (Fig. 2b). Forskolin was also able to augment insulin secretion at $8 \mathrm{mmol} / \mathrm{l}$ glucose in ZL islets, albeit to much lower absolute levels than in ZF islets (30 and $21 \%$ of secretion in $\mathrm{ZF}$ islets in the absence and presence of palmitate, respectively) (Fig. 2b). These results support an enhanced upregulatory effect of NEFA in ZF islets on multiple stimuli for insulin secretion.

Fatty acid partitioning into oxidation and esterification pathways is increased in ZF islets

Palmitate oxidation was reduced at high glucose (16 mmol/1) compared to low glucose $(3 \mathrm{mmol} / 1)$, but for these glucose concentrations was not different between $\mathrm{ZF}$ and ZL islets (Fig. 3a). Palmitate oxidation at $8 \mathrm{mmol} / \mathrm{l}$ glucose, however, was $89 \%$ higher in ZF compared with ZL islets (Fig. 3a). This result is consistent with less suppression of fatty acid oxidation in ZF islets by an increase in glucose concentration within the physiological range. The net
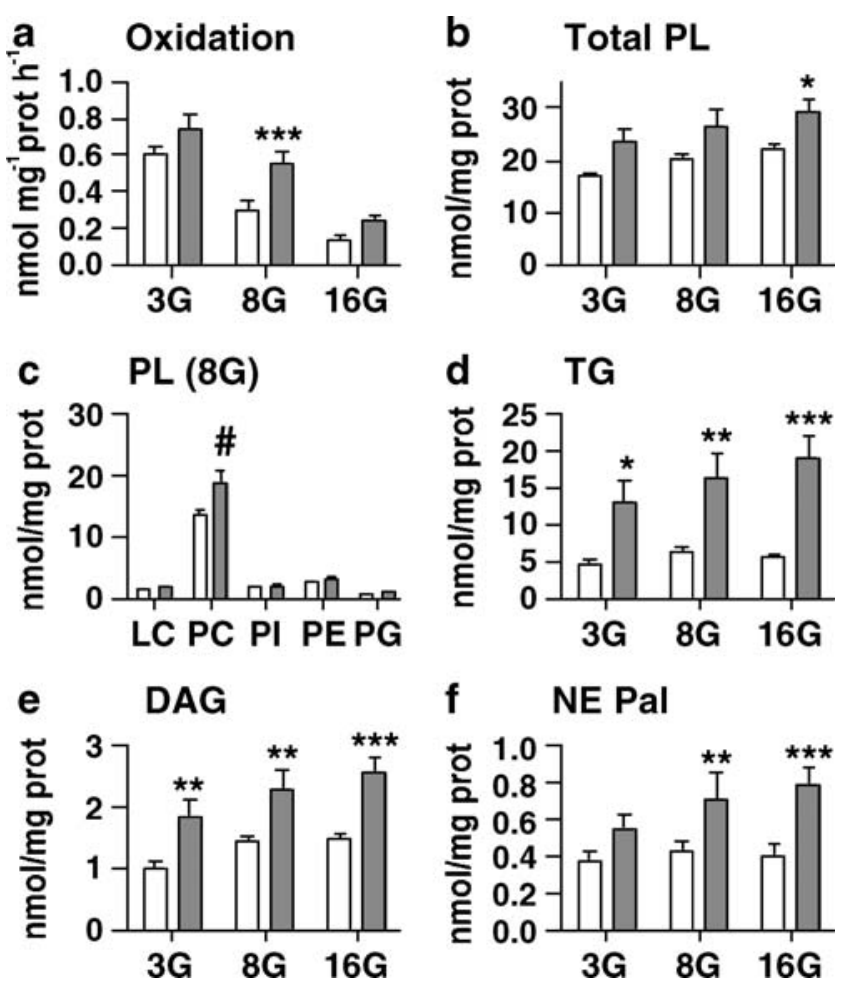

f NE Pal

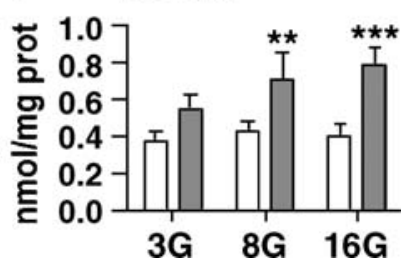

Fig. 3 Palmitate oxidation at intermediate glucose levels and net palmitate esterification into complex lipids are increased in ZF islets. a Palmitate oxidation. Islets from ZF (grey bars) and ZL (white bars) rats were prelabelled for $40 \mathrm{~h}$ with $\left[9,10(\mathrm{n})-{ }^{3} \mathrm{H}\right]$ palmitate and incubated for $2 \mathrm{~h}$ in $\left[9,10(\mathrm{n})-{ }^{3} \mathrm{H}\right]$ palmitate oxidation media at 3 $(3 \mathrm{G}), 8(8 \mathrm{G})$ and $16(16 \mathrm{G}) \mathrm{mmol} / \mathrm{l}$ glucose. b-f Islets were cultured for $40 \mathrm{~h}$ in $\left[1-{ }^{14} \mathrm{C}\right]$ palmitate esterification media at 3,8 and $16 \mathrm{mmol} /$ 1 glucose. Esterification into total phospholipids (b), phospholipid species lysophosphatidylcholine (LC), phosphatidylcholine (PC), phosphatidylinositol (PI), phosphatidylethanolamine (PE) and phosphatidylglycerol (PG) (c), triglyceride (d) and diacylglycerol (e). f Determination of islet non-esterified palmitate content at time $40 \mathrm{~h}$. Means \pm SEM of six determinations from islets of six rats of each genotype in two separate experiments. Two-way ANOVA post hoc analyses: ${ }^{*} p<0.05,{ }^{* *} p<0.01,{ }^{* * *} p<0.001$. Student's $t$-test: $\# p<0.05$

esterification of palmitate into total complex lipids (total esterification minus lipolysis) was 65,53 and $71 \%$ higher at 3,8 and $16 \mathrm{mmol} / 1$ glucose, respectively (not shown); esterification into TG $(169,164$ and $229 \%$ higher in ZF) (Fig. 3d) and diacylglycerol (81, 54 and 70\% higher in ZF) (Fig. 3e) contributed mostly to this difference. Net esterification into total phospholipids was minimally increased in ZF compared with ZL islets (Fig. 3b) and this was predominantly due to increased esterification into phosphatidylcholine rather than other phospholipid species (Fig. 3c). Interestingly, elevated glucose resulted in higher levels of non-esterified palmitate (an index of cellular LCCoA [13]) in ZF compared with ZL islets (66 and 98\% higher than $\mathrm{ZL}$ at 8 and $16 \mathrm{mmol} / 1$ glucose, respectively) (Fig. 3f). 
Glucose-stimulated lipolysis is enhanced in ZF islets

Basal lipolysis was 2-fold higher in ZF compared with ZL islets (Fig. 4a,b). Of particular interest, an increase in glucose concentration increased lipolysis by $65 \%$ in $\mathrm{ZL}$ islets and by $161 \%$ in $\mathrm{ZF}$ islets, such that at high glucose, lipolysis was 3.2-fold higher in ZF compared with ZL islets (Fig. 4a). GLP-1 and forskolin, both of which increase the activity of cyclic AMP-dependent protein kinase (PKA), failed to increase lipolysis in either $\mathrm{ZF}$ or $\mathrm{ZL}$ islets (Fig. 4b). In concordance with the increased basal lipolysis in $\mathrm{ZF}$ islets, the lipolytic enzyme activities of NCEH and TGL were 148 and $121 \%$ higher, respectively, in ZF compared with ZL islets (Fig. 4c,d). Hormone-sensitive lipase $(H s l)$ mRNA was measured in $\mathrm{ZL}$ and $\mathrm{ZF}$ islets by semiquantitative RT-PCR. There was a trend for increased $H s l$ mRNA expression in $\mathrm{ZF}$ islets, but this did not reach statistical significance (Fig. 4e).
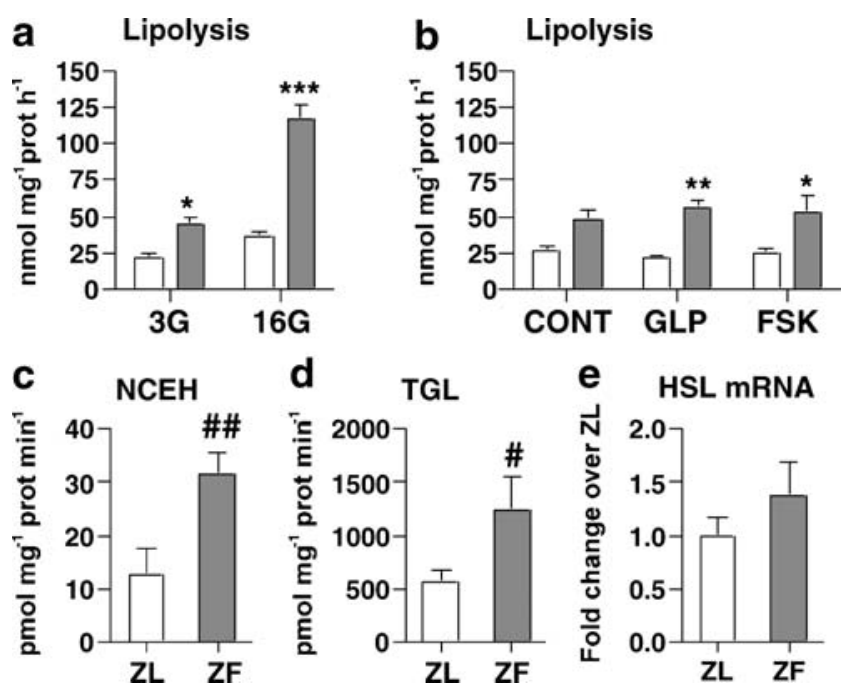

Fig. 4 Glucose-stimulated lipolysis is increased in islets of ZF rats. a, b Lipolysis measured in islets from ZF (grey bars) and ZL (white bars) rats as the rate of glycerol release at (a) $3(3 \mathrm{G})$ and $16 \mathrm{mmol} / \mathrm{l}$ (16G) glucose and (b) at $3 \mathrm{mmol} / \mathrm{l}$ glucose in the absence or presence of $10 \mathrm{nmol} / 1 \mathrm{GLP}-1$ or $5 \mu \mathrm{mol} / 1$ forskolin (FSK). Means \pm SEM of ten determinations from islets of ten rats in five separate experiments (a) and six determinations from islets of six rats from three separate experiments (b). Two-way ANOVA post hoc analyses: ${ }^{*} p<0.05, \quad * * p<0.01, * * * p<0.001$ compared with ZL for same condition. c, d Neutral cholesterol ester hydrolase and triglyceride lipase activities, respectively, measured from islet cell extracts. Means \pm SEM of four to six determinations from islets from $8-12$ rats of each genotype in three separate experiments. Student's $t$-test: $\# p<0.05, \# \# p<0.01$ compared with ZL. e Hsl mRNA expression by semiquantitative RT-PCR with correction for $\beta$-actin. Means \pm SEM of islets from 11-12 rats of each genotype
Lipolysis inhibition by Orlistat abolishes glucosestimulated lipolysis and markedly reduces insulin secretion in $\mathrm{ZF}$ islets

Orlistat binds to the catalytic site of numerous lipases, including HSL [17, 22]. Previously, inhibition of lipolysis by Orlistat in normal rat islets has been shown to inhibit GSIS, suggesting a role for lipolysis in normal insulin secretion [17]. To determine whether the enhanced glucosestimulated lipolysis observed in ZF islets could be involved in islet compensatory processes for insulin resistance, lipolysis and NEFA-augmented GSIS were measured in $\mathrm{ZF}$ islets in the presence and absence of Orlistat. Orlistat had no effect on basal lipolysis, but completely abolished glucose-stimulated lipolysis in the ZF islets (Fig. 5a). Lipolysis inhibition by Orlistat reduced NEFA-augmented GSIS by $53 \%$ (Fig. 5b).

Expression of key transcription factors and enzymes of lipid metabolism in ZL and ZF islets

The expression of the insulin gene at the mRNA level was significantly increased by $49 \%$ in ZF compared with ZL
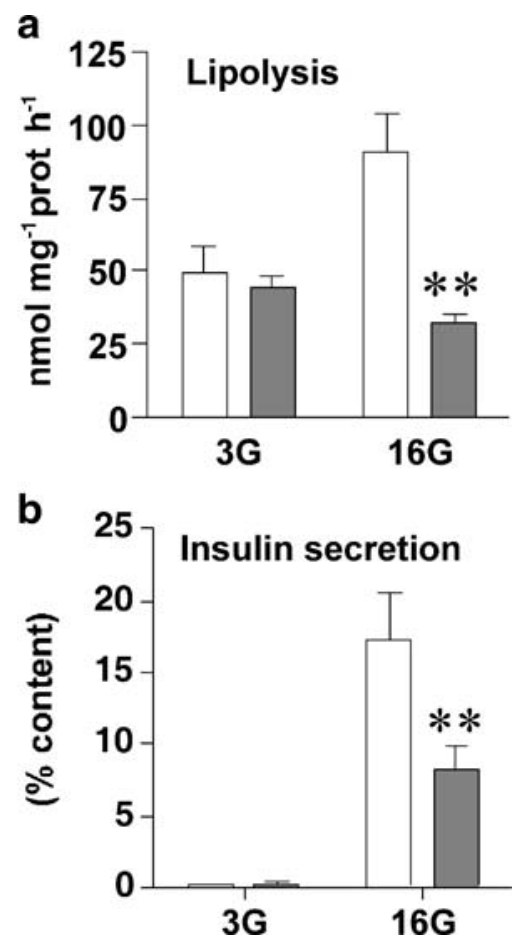

Fig. 5 Inhibition of lipolysis impairs glucose-stimulated insulin secretion from ZF islets. a, b Lipolysis and insulin secretion respectively were measured in media containing $0.25 \mathrm{mmol} / 1$ palmitate at $3(3 \mathrm{G})$ and $16(16 \mathrm{G}) \mathrm{mmol} / \mathrm{l}$ glucose in the absence (white bars) or presence (grey bars) of $0.2 \mathrm{mmol} / 1$ Orlistat. Means $\pm \mathrm{SEM}$ of four (a) or eight (b) determinations from islets of eight animals of each genotype in two separate experiments. Two-way ANOVA: Orlistat effect on lipolysis, $p<0.01$; Orlistat effect on insulin secretion, $p<0.05$. Bonferroni post hoc test: ${ }^{* *} p<0.01$ 
islets (Fig. 6). Of the lipid metabolism transcription factors (peroxisomal proliferator-activated receptors $\alpha, \beta$ and $\gamma$ [Ppara, Pparb, Pparg] and sterol-regulatory element binding transcription factor 1c [Srebflc]) and the transcription factor coactivator peroxisomal proliferator-activated receptor $\gamma$ coactivator $1 \alpha$ (Ppargcla), only Ppara (reduced by $31 \%$ ) and Pparg (increased by $28 \%$ ) mRNAs showed changes in ZF islets (Fig. 6). Expression levels of free fatty acid receptor 1 (Ffarl; also known as the G-protein receptor 40 [Gpr40]) and $C d 36$ (fatty acid transport protein) were not altered (Fig. 6). Of mRNAs for the lipid metabolism enzymes that favour fatty acid oxidation (Cpt1, acyl-CoA oxidase, uncoupling protein 2 and malonyl-CoA decarboxylase), only Cpt 1 mRNA was altered in $\mathrm{ZF}$ islets (increased by $51 \%$ ). Of mRNAs for

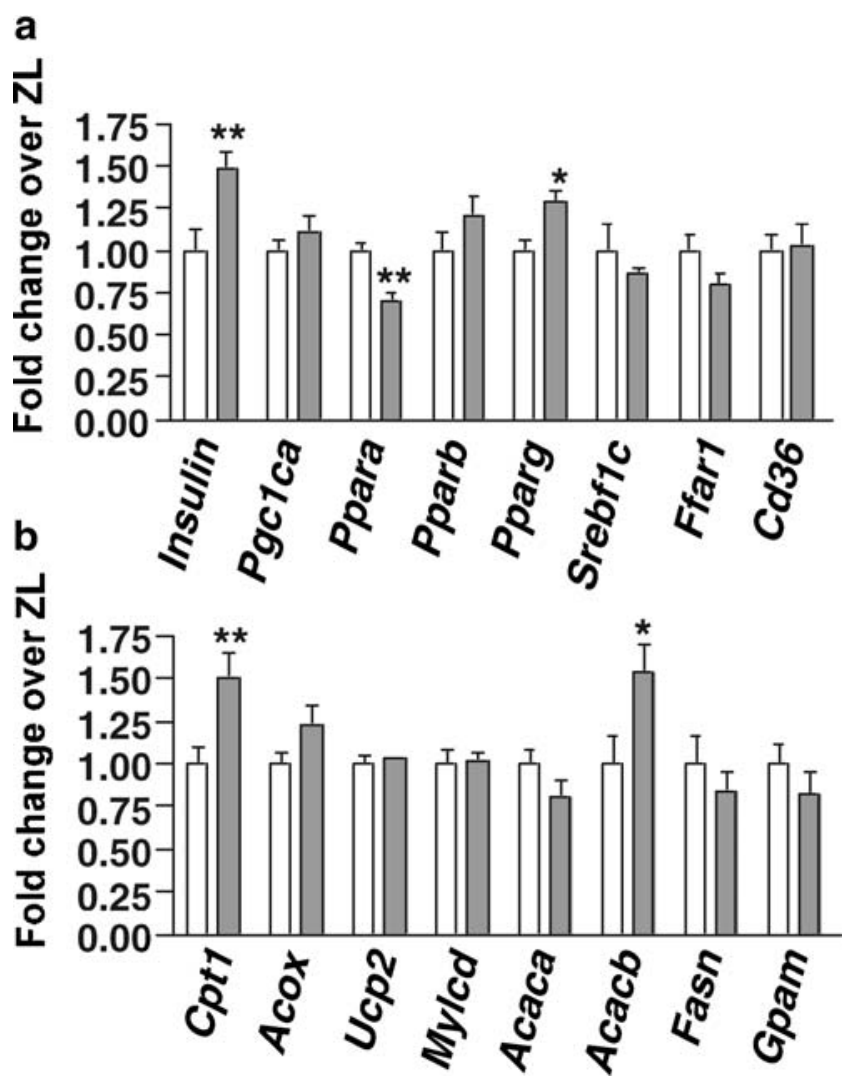

Fig. 6 Expression of genes related to lipid metabolism and signalling at the mRNA level in $\mathrm{ZF}$ and $\mathrm{ZL}$ islets. Insulin, peroxisomal proliferator-activated receptor $\gamma$ coactivator $1 \alpha(P g c l c a)$, peroxisomal proliferator-activated receptors $\alpha$ (Ppara), $\beta$ (Pparb) and $\gamma$ (Pparg), sterol regulatory element binding transcription factor 1c (Srebflc), free fatty acid receptor 1 (Ffarl), CD 36 (a), carnitine palmitoyltransferase 1 (Cpt1), acyl-CoA oxidase (Acox), uncoupling protein 2 (Ucp2), malonyl-CoA decarboxylase $(M l y c d)$, acetyl-CoA carboxylases $\alpha$ (Acaca) and $\beta$ (Acacb), fatty acid synthase (Fasn) and mitochondrial glycerol-3-phosphate acyltransferase (Gpam) (b) mRNA levels were measured in ZF (grey bars) and ZL (white bars) islets by RT-PCR analysis followed by Southern blot hybridisation and autoradiography. Expression was corrected for $\beta$-actin mRNA levels. Means \pm SEM of islets from $5-11$ rats of each genotype. Student's $t$-test: ${ }^{*} p<0.05$, $* * p<0.01$ enzymes favouring fatty acid synthesis and esterification processes (acetyl-CoA carboxylases $\alpha$ and $\beta$ [Acaca and $A c a c b]$, fatty acid synthase [Fasn] and mitochondrial glycerol-3-phosphate acyltransferase [Gpam]), only Acacb mRNA (the mitochondrial isoform of acetyl-CoA carboxylase) was increased in $\mathrm{ZF}$ compared with $\mathrm{ZL}$ islets (increased by $54 \%$ ) (Fig. 6).

\section{Discussion}

The findings of this study support an important role for lipid signalling (activation of the NEFA receptor FFAR1 and the production of lipid-derived signals, such as diacylglycerol, signalling phospholipids or fatty acyl-CoA, that are known to modulate biological effectors such as ion channels and enzymes) in beta-cell compensation for insulin resistance in $\mathrm{ZF}$ rats. A key observation in this regard was that the normal augmentation of GSIS by exogenous palmitate was markedly enhanced in ZF compared with ZL islets, and this effect was particularly apparent within the physiological range of plasma glucose (5-8 mmol/l). Also, the incretin effect of GLP-1 and the insulin response to the cyclic AMP-enhancing agent forskolin were both further augmented by exogenous palmitate at $8 \mathrm{mmol} / \mathrm{l}$ glucose in $\mathrm{ZF}$ islets. In investigating the underlying mechanism of this NEFA effect, we found that all pathways of fatty acid partitioning (fatty acid oxidation, esterification and lipolysis) were enhanced in ZF islets. Importantly, the lipase inhibitor Orlistat reversed the enhanced GSIS in ZF islets. Also, our results provide potential insight into the regulation of lipolysis in $\mathrm{ZF}$ islets by showing increased activities of NCEH and TGL. Together, these findings support enhancement in ZF islets of the normal cycle of TG production and lipolysis as a mechanism of their insulin hypersecretion. In addition, enhanced lipolysis may prevent lipid-induced islet damage by minimising the accumulation of toxic lipids. Our results thus support a beneficial role for the in vivo hyperlipidaemia in $\mathrm{ZF}$ rats in the beta cell compensation for insulin resistance, as opposed to the belief of some that chronic excess of NEFA, even in the absence of hyperglycaemia, invariably causes beta cell dysfunction or death $[6,7]$.

The finding that NEFA dramatically synergised with GLP-1 and the cyclic AMP-increasing agent forskolin in augmenting GSIS in ZF islets is interesting with respect to beta cell compensation. This finding is in keeping with the previous observation that NEFA augment insulin secretion in response not only to glucose but also to all fuel and nonfuel secretagogues, including GLP-1 [13]. The present findings reinforce the concept that NEFA are not only essential for insulin secretion [23] but are also involved in enhancement of secretion in response to a wide variety of 
secretagogues. Consequently, NEFA and circulating TG within lipoproteins, which are often elevated in obesityassociated insulin resistance states [24], are prime candidates for factors that link visceral adiposity and beta cell compensation for insulin resistance [25]. Interestingly, GLP-1 levels were reported to be increased in the highfat-fed dog model of insulin resistance [26], also underscoring the potential role of GLP-1 and NEFA synergy in hyperinsulinaemic compensation mechanisms.

Even though we have shown coordinately increased fatty acid esterification and lipolysis processes in response to glucose in ZF islets, we have not proven that TG-FA cycling exists as we have not shown that the NEFA resulting from lipolysis are in fact re-esterified. However, it seems likely that at least some of the cytosolic NEFA from lipolysis would be re-activated to acyl-CoA, followed by either re-esterification (cycling) or oxidation. Intracellular TG-FA cycling is known to occur in multiple other tissues, including adipose tissue [27], muscle [28] and liver (reviewed in [29]). It is a new concept to suggest a role for TG-FA cycling in beta cell metabolic signal transduction and compensation for insulin resistance. We propose a model (Fig. 7) in which glucose promotes activity in a beta cell TG-FA cycle by (1) the malonyl-CoA/LC-CoA switch of fatty metabolism from oxidation to esterification [3, 13]; (2) providing glycerol-3-phosphate (Gly-3-P) for synthesis of complex glycerolipids; and (3) activation of lipolysis, as demonstrated in this study. NEFA also contribute to activation of the cycle by providing the other substrate
(LC-CoA) for condensation with Gly-3-P. Thus, elevated glucose, in the presence of NEFA, will result in increased cycle intermediates, such as unesterified fatty acid, LC$\mathrm{CoA}$ and diacylglycerol, that have lipid-signalling potential for augmentation of glucose-stimulated insulin exocytosis $[3,13,30]$. In agreement with this schema, the increased glucose metabolism and anaplerosis that we observed previously in ZF islets [8] will favour activity in the cycle, as will the finding in this study of increased TGL activity. Also, we showed previously that activity of mitochondrial Gly-3-P dehydrogenase (mGPD), the rate-limiting enzyme of the Gly-3-P shunt, was reduced by $50 \%$ in ZF islets [8], which should increase the availability of Gly-3-P for glycerolipid synthesis.

Increased glucose metabolism and increased availability of NEFA, resulting in increased TG synthesis, are almost certainly contributors to the proposed TG-FA cycle in ZF islets (Fig. 7). The regulatory factors of cycling at the level of lipase enzymes in both normal and compensating islets, however, are yet to be determined. ZF islet extracts were shown to have greater than 2-fold increased TGL activity, which could be a result of increased expression of lipase enzymes or increased lipase intrinsic activity. There was a non-significant $38 \%$ increase in $H s l$ mRNA levels. Interestingly, we have now analysed islet $H s l$ mRNA levels by realtime PCR in a new series of ZL and ZF animals that had a sham laparotomy for another study protocol. In that study, $H s l$ mRNA was significantly increased in $\mathrm{ZF}$ islets $(1.49 \pm$ 0.08 compared with $0.78 \pm 0.11$ relative units; ZF vs ZL,

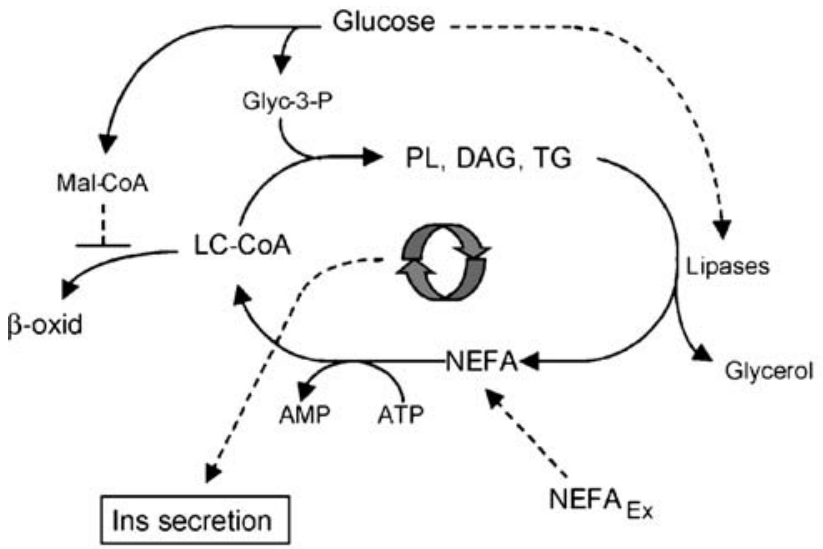

Zucker Lean

Fig. 7 Model depicting the islet TG-FA cycle in control ZL islets and its role in beta cell compensation in ZF islets. An elevation in glucose promotes the cycle by (1) inhibition of fatty acid $\beta$-oxidation via malonyl-CoA such that cytosolic LC-CoA increases; (2) provision of glycerol-3-phosphate (Gly-3-P) for glycerolipid synthesis; and (3) activation of lipolysis. Exogenous NEFA $\left(\mathrm{NEFA}_{\mathrm{Ex}}\right)$ feeds into the cycle, increasing the levels of several intermediates. Increased activity of this cycle in $\mathrm{ZF}$ islets, via increased glucose metabolism providing

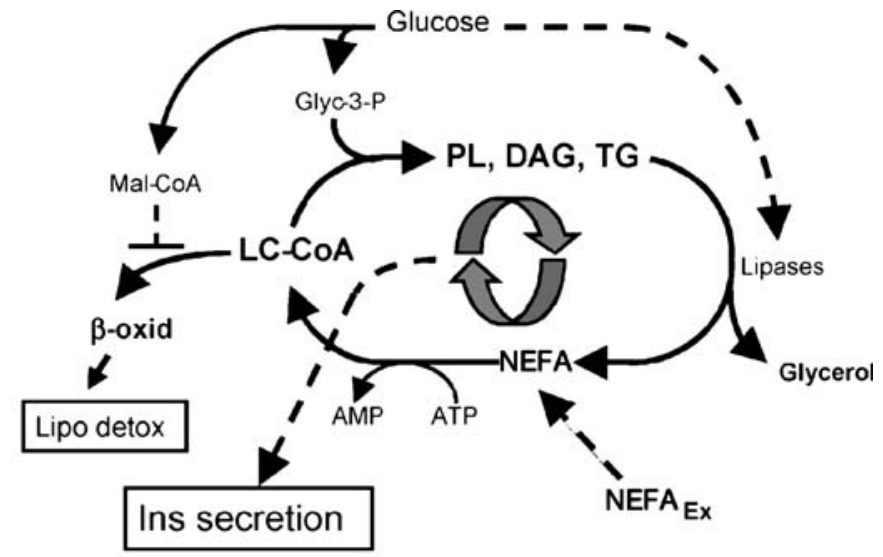

Zucker Fatty

more Gly-3-P and higher malonyl-CoA levels, a greater supply of exogenous NEFA and higher activity of lipases, contributes to beta cell compensation for insulin resistance through the provision of increased lipid signalling molecules for exocytosis, such as LC-CoA and diacylglycerol DAG. The cycle also allows the detoxification of lipids via lipolysis and the enhanced $\beta$-oxidation of fatty acids. The TG-FA cycle may also protect the beta cell due to the fact that it is a futile cycle resulting in the consumption of ATP 
$p<0.01)$. Therefore, it seems likely that the expression of $H s l$ is higher in ZF islets, which is consistent with the enzyme activity data. The role of HSL in islet beta cell function is unclear, however, as insulin secretion has been shown to be little or not affected in $H s l-/-$ animals $[18,31]$. We showed impaired GSIS in only male fasted $\mathrm{Hsl}-/-$ mice, and this was completely reversed by the provision of exogenous NEFA [18]. The expression of HSL in beta cells, however, has been shown to be upregulated by a prolonged high glucose concentration [32] and HSL has been shown to be co-localised with insulin granules [33]. There has been particular interest in the role of non-HSL lipases in adipose tissue metabolism $[34,35]$. The roles of these alternative lipases in the islet also warrant investigation.

There is increasing evidence that islet beta cell lipolysis is increased by elevated glucose, as shown previously in normal rat islets [17] and in both wild-type and $H s l-1-$ mouse islets [18]. We have also shown glucose-stimulated lipolysis to be tightly correlated with GSIS in INS832/13 beta cells (C. J. Nolan and M. Prentki, unpublished observations). It may be that the effect of glucose on lipolysis results from its effect in increasing TG synthesis, perhaps into a labile intracellular pool, thus providing more substrate for lipase enzymes. Another possibility is that elevated glucose might activate lipase enzyme activity. Of relevance, we recently showed that LC-CoA, which is thought to increase in the cytoplasm in response to elevated glucose $[3,13]$, activates lipolysis in vitro in islet extracts from control and $\mathrm{Hsl}-/-$ mice [36]. It is an attractive hypothesis, therefore, that activation of lipolysis by LCCoA could also be involved in the enhanced TG-FA cycling that was observed in $\mathrm{ZF}$ islets.

In fat cells, hormonal stimulation of lipolysis is by activation of HSL via PKA phosphorylation. While GLP-1 and forskolin, both activators of PKA, have been shown to increase lipolysis in clonal pancreatic beta cells (HIT cells) [37], neither agent enhanced lipolysis in ZF or ZL islets. This finding is in agreement with our study of $\mathrm{Hsl}$ wildtype and knockout mice [18]. Glucose activation of lipolysis was noted in the HSL-deficient mice but no significant effect of GLP-1 on lipolysis was observed [18]. Thus, it seems that glucose acts via a mechanism other than PKA, possibly via an enzyme other than HSL [18]. The results also indicate that the synergistic effect of GLP-1 or forskolin with NEFA in augmenting GSIS is not due to an effect of GLP-1 or forskolin in accelerating lipolysis.

At first glance, it seems odd that increased malonyl$\mathrm{CoA} / \mathrm{LC}-\mathrm{CoA}$ signalling and increased fatty acid oxidation at a physiologically relevant glucose level should occur together in ZF islets. Interestingly, the expression of important enzymes of both pathways, ACACB (synthesises malonyl-CoA) and CPT1 (the rate-limiting step in mitochondrial fatty acid oxidation), were increased in $\mathrm{ZF}$ islets.
We suspect that the increased fatty acid oxidation is maintained, despite more inhibition of CPT1 by malonylCoA, because of the maintenance of higher levels of LCCoA (substrate for fatty acid oxidation) by accelerated TG-FA cycling. Previously it has been shown that inhibition of fatty acid oxidation by an inhibitor of CPT1 reduces basal hypersecretion in ZDF rats, such that the maintenance of fatty acid oxidation may also be important in compensation processes [38]. Islets of the $\mathrm{ZF}$ rat displayed none of the well-described features of lipotoxicity [7, 39-43]. The TG content was mildly decreased rather than dramatically increased [7, 40]. The mRNA expression of genes that promote lipogenesis and fatty acid esterification (Srebflc, Acaca, Fasn and Gpam) was not altered in $\mathrm{ZF}$ islets. Thus, the question arises as to how ZF islets escape lipotoxicity. As illustrated in Fig. 7, enhanced TGFA cycling provides an attractive mechanism of protection from lipid toxicity in the face of obesity-associated hyperlipidaemia. The cycle prevents steatosis due to the high rates of TG lipolysis and by allowing the maintenance of LC-CoA as a substrate for detoxification via fatty acid oxidation. In addition, TG-FA cycling is futile, in that fatty acid activation to LC-CoA and its metabolism back to NEFA via TG expend ATP energy.

In conclusion, our results document very active fatty acid glucose-responsive esterification/lipolysis processes in pancreatic beta cells, which are further enhanced in the islets of ZF rats. We [16, 18] and co-workers [17] have provided genetic ( $\mathrm{Hsl}$ knockout studies) and pharmacological (Orlistat, dimethylpyrazole) evidence that lipolysis plays a role in GSIS. This study expands that concept by providing evidence that enhanced lipolysis and, most probably, TG-FA cycling in ZF islets appear to be a mechanism for the enhanced insulin secretion that characterises beta cell compensation for their insulin resistance.

Acknowledgements We wish to thank Y. Long and J. Morin for expert technical assistance and B. E. Corkey for thoughtful discussion of the work. This work was supported by grants from the American Diabetes Association (J. L. Leahy), the National Institute of Health (J. L. Leahy, DK56818; M. Prentki, DK-63356), Genome Quebec, Canada (M. Prentki) and the Canadian Institute of Health Research (CIHR) (M. Prentki). C. Guay is supported by a graduate studentship from the Fonds de Recherche en Santé du Québec.

\section{References}

1. Kahn SE, Prigeon RL, McCulloch DK et al (1993) Quantification of the relationship between insulin sensitivity and beta-cell function in human subjects. Evidence for a hyperbolic function. Diabetes 42:1663-1672

2. Shulman GI (2000) Cellular mechanisms of insulin resistance. J Clin Invest 106:171-176

3. Prentki M, Joly E, El-Assaad W, Roduit R (2002) Malonyl-CoA signaling, lipid partitioning, and glucolipotoxicity: role in beta- 
cell adaptation and failure in the etiology of diabetes. Diabetes 51 (Suppl 3):S405-S413

4. Porte D (2001) Clinical importance of insulin secretion and its interaction with insulin resistance in the treatment of type 2 diabetes mellitus and its complications. Diabetes Metab Res Rev 17:181-188

5. Crespin SR, Greenough WB 3rd, Steinberg D (1969) Stimulation of insulin secretion by infusion of free fatty acids. J Clin Invest 48:1934-1943

6. McGarry JD (2002) Banting lecture 2001: dysregulation of fatty acid metabolism in the etiology of type 2 diabetes. Diabetes 51:7-18

7. Lee Y, Hirose H, Ohneda M, Johnson JH, McGarry JD, Unger RH (1994) Beta-cell lipotoxicity in the pathogenesis of noninsulin-dependent diabetes mellitus of obese rats: impairment in adipocyte-beta-cell relationships. Proc Natl Acad Sci USA 91:10878-10882

8. Liu YQ, Jetton TL, Leahy JL (2002) Beta-cell adaptation to insulin resistance. Increased pyruvate carboxylase and malatepyruvate shuttle activity in islets of nondiabetic Zucker fatty rats. J Biol Chem 277:39163-39168

9. Cockburn BN, Ostrega DM, Sturis J, Kubstrup C, Polonsky KS, Bell GI (1997) Changes in pancreatic islet glucokinase and hexokinase activities with increasing age, obesity, and the onset of diabetes. Diabetes 46:1434-1439

10. Pick A, Clark J, Kubstrup C et al (1998) Role of apoptosis in failure of beta-cell mass compensation for insulin resistance and beta-cell defects in the male Zucker diabetic fatty rat. Diabetes 47:358-364

11. Prentki M (1996) New insights into pancreatic beta-cell metabolic signaling in insulin secretion. Eur J Endocrinol 134:272-286

12. Prentki M, Vischer S, Glennon MC, Regazzi R, Deeney JT, Corkey BE (1992) Malonyl-CoA and long chain acyl-CoA esters as metabolic coupling factors in nutrient-induced insulin secretion. J Biol Chem 267:5802-5810

13. Roduit R, Nolan C, Alarcon C et al (2004) A role for the malonylCoA/long-chain acyl-CoA pathway of lipid signaling in the regulation of insulin secretion in response to both fuel and nonfuel stimuli. Diabetes 53:1007-1019

14. McGarry JD, Brown NF (1997) The mitochondrial carnitine palmitoyltransferase system. From concept to molecular analysis. Eur J Biochem 244:1-14

15. Ruderman N, Prentki M (2004) AMP kinase and malonyl-CoA: targets for therapy of the metabolic syndrome. Nat Rev Drug Discov 3:340-351

16. Masiello P, Novelli M, Bombara M et al (2002) The antilipolytic agent 3,5-dimethylpyrazole inhibits insulin release in response to both nutrient secretagogues and cyclic adenosine monophosphate agonists in isolated rat islets. Metabolism 51:110-114

17. Mulder H, Yang S, Winzell MS, Holm C, Ahren B (2004) Inhibition of lipase activity and lipolysis in rat islets reduces insulin secretion. Diabetes 53:122-128

18. Peyot ML, Nolan CJ, Soni K et al (2004) Hormone-sensitive lipase has a role in lipid signaling for insulin secretion but is nonessential for the incretin action of glucagon-like Peptide 1 . Diabetes 53:1733-1742

19. Winzell MS, Svensson H, Enerback S et al (2003) Pancreatic betacell lipotoxicity induced by overexpression of hormone-sensitive lipase. Diabetes 52:2057-2065

20. El-Assaad W, Buteau J, Peyot ML et al (2003) Saturated fatty acids synergize with elevated glucose to cause pancreatic beta-cell death. Endocrinology 144:4154-4163

21. Gremlich S, Nolan C, Roduit R et al (2005) Pancreatic islet adaptation to fasting is dependent on peroxisome proliferatoractivated receptor alpha transcriptional up-regulation of fatty acid oxidation. Endocrinology 146:375-382
22. Smith GM, Garton AJ, Aitken A, Yeaman SJ (1996) Evidence for a multi-domain structure for hormone-sensitive lipase. FEBS Lett 396:90-94

23. Dobbins RL, Chester MW, Stevenson BE, Daniels MB, Stein DT, McGarry JD (1998) A fatty acid-dependent step is critically important for both glucose- and non-glucose-stimulated insulin secretion. J Clin Invest 101:2370-2376

24. Moller DE, Kaufman KD (2005) Metabolic syndrome: a clinical and molecular perspective. Annu Rev Med 56:45-62

25. Cruz ML, Bergman RN, Goran MI (2002) Unique effect of visceral fat on insulin sensitivity in obese Hispanic children with a family history of type 2 diabetes. Diabetes Care 25:1631-1636

26. van Citters GW, Kabir M, Kim SP et al (2002) Elevated glucagonlike peptide-1-(7-36)-amide, but not glucose, associated with hyperinsulinemic compensation for fat feeding. J Clin Endocrinol Metab 87:5191-5198

27. Reshef L, Olswang Y, Cassuto H et al (2003) Glyceroneogenesis and the triglyceride/fatty acid cycle. J Biol Chem 278: 30413-30416

28. Bastie CC, Hajri T, Drover VA, Grimaldi PA, Abumrad NA (2004) CD36 in myocytes channels fatty acids to a lipaseaccessible triglyceride pool that is related to cell lipid and insulin responsiveness. Diabetes 53:2209-2216

29. Murphy DJ (2001) The biogenesis and functions of lipid bodies in animals, plants and microorganisms. Prog Lipid Res 40:325-438

30. Prentki M, Corkey BE (1996) Are the beta-cell signaling molecules malonyl-CoA and cystolic long-chain acyl-CoA implicated in multiple tissue defects of obesity and NIDDM? Diabetes 45:273-283

31. Mulder H, Sorhede-Winzell M, Contreras JA et al (2003) Hormone-sensitive lipase null mice exhibit signs of impaired insulin sensitivity whereas insulin secretion is intact. J Biol Chem 278:36380-36388

32. Winzell MS, Svensson H, Arner P, Ahren B, Holm C (2001) The expression of hormone-sensitive lipase in clonal beta-cells and rat islets is induced by long-term exposure to high glucose. Diabetes 50:2225-2230

33. Lindvall H, Nevsten P, Strom K et al (2004) A novel hormonesensitive lipase isoform expressed in pancreatic beta-cells. J Biol Chem 279:3828-3836

34. Soni KG, Lehner R, Metalnikov P et al (2004) Carboxylesterase 3 (EC 3.1.1.1) is a major adipocyte lipase. J Biol Chem 279: 40683-40689

35. Zechner R, Strauss JG, Haemmerle G, Lass A, Zimmermann R (2005) Lipolysis: pathway under construction. Curr Opin Lipidol $16: 333-340$

36. Hu L, Deeney JT, Nolan CJ et al (2005) Regulation of lipolytic activity by long-chain acyl-coenzyme a in islets and adipocytes. Am J Physiol Endocrinol Metab 289:1085-1092

37. Yaney GC, Civelek VN, Richard AM et al (2001) Glucagon-like peptide 1 stimulates lipolysis in clonal pancreatic beta-cells (HIT). Diabetes 50:56-62

38. Zhou YP, Cockburn BN, Pugh W, Polonsky KS (1999) Basal insulin hypersecretion in insulin-resistant Zucker diabetic and Zucker fatty rats: role of enhanced fuel metabolism. Metabolism 48:857-864

39. Kakuma T, Lee Y, Higa M et al (2000) Leptin, troglitazone, and the expression of sterol regulatory element binding proteins in liver and pancreatic islets. Proc Natl Acad Sci USA 97:8536-8541

40. Lee Y, Hirose H, Zhou YT, Esser V, McGarry JD, Unger RH (1997) Increased lipogenic capacity of the islets of obese rats: a role in the pathogenesis of NIDDM. Diabetes 46:408-413 
41. Zhou YT, Shimabukuro M, Lee Y et al (1998) Enhanced de novo lipogenesis in the leptin-unresponsive pancreatic islets of prediabetic Zucker diabetic fatty rats: role in the pathogenesis of lipotoxic diabetes. Diabetes 47:1904-1908

42. Hirose H, Lee YH, Inman LR, Nagasawa Y, Johnson JH, Unger RH (1996) Defective fatty acid-mediated beta-cell compensation in Zucker diabetic fatty rats. Pathogenic implications for obesitydependent diabetes. J Biol Chem 271:5633-5637

43. Harmon JS, Gleason CE, Tanaka Y, Poitout V, Robertson RP (2001) Antecedent hyperglycemia, not hyperlipidemia, is associated with increased islet triacylglycerol content and decreased insulin gene mRNA level in Zucker diabetic fatty rats. Diabetes 50:2481-2486 